\title{
Controllable morphology evolution of mesoporous silica FDU-12 via a simple route
}

\author{
Jia-Yue WANG ${ }^{1}$, Peng Du ${ }^{1,}{ }^{\dagger}$, Xi-Long WANG $^{1}$, Ke-Bin $C H I^{2 *}$, Ai-Jun DUAN ${ }^{1 *}$ \\ and Zhen $\mathrm{ZHAO}^{1 *}$ \\ ${ }^{1}$ China University of Petroleum, Beijing 102249, P. R. China \\ ${ }^{2}$ Petrochemical Research Institute, PetroChina Company Limited, Beijing 100195, P. R. China \\ *Corresponding author: duanaijun@cup.edu.cn; zhenzhao@cup.edu.cn
}

This author has equal contribution as the first author.

Keywords: FDU-12, morphology, water-bath temperature.

Abstract. Mesoporous silica FDU-12 with different morphologies has been successfully hydrothermal synthesized under static or stirring conditions by changing the water-bath temperature. The as-synthesized materials were characterized by X-ray powder diffraction(XRD), $\mathrm{N}_{2}$ adsorption-desorption, scanning electron microscopy (SEM) and transmission electron microscopy (TEM). The SEM micrograph showed that the FDU-12 morphologies kept regular hexagonal prism under stirring condition, while under static condition those samples obtained exhibited the morphologies from sphere to different polyhedrons by changing the water-bath temperature.

\section{Introduction}

Mesoporous silicas have shown potential applications in catalytic reaction, adsorption, separation, biotechnology and pharmacy due to their highly ordered pore structures, adjustable pore size, large surface areas and huge pore volume. In addition to these physical properties, the morphology of the material, however, had a great effect on the applications in different areas. Till now, there have also been several reports that describe the methods to control the morphology of the mesoporous particles. Mesoporous silica SBA-15 molecular sieves were synthesized by Ding[1] using hydrothermal method with the changes of $\mathrm{H}_{2} \mathrm{SO}_{4}$ concentration from 0.1 to $2.3 \mathrm{~mol} / \mathrm{L}$. The results showed that the length of fries-like agglomerate particle units decreased from 3 to $0.5 \mu \mathrm{m}$ with $\mathrm{H}_{2} \mathrm{SO}_{4}$ concentration of 0.2 to $2.3 \mathrm{~mol} / \mathrm{L}$. Zhou[2] found that crystallization temperature was a key factor that had great effects on the morphology of SAB-15. The SEM images revealed that the morphology of SBA-15 was changed from "wheat-like" agglomerate particles to "banana-like" short rods with the increasing of the crystallization temperature. FDU-12 mesoporous silicas with highly ordered face-centred cubic (Fm3m) structure were firstly synthesized using F127 as a template under strong acidic conditions[3]. Due to their large surface area and uniform and adjustable pore size, the materials have received much attention. But few work has been done about discussing their morphology changes. In this research, FDU-12 with different morphologies was hydrothermally synthesized under static or stirring condition at different water-bath temperatures.

\section{Experimental section}

\section{Synthesis}

The synthesis procedure follows: $2.0 \mathrm{~g}$ of triblock copolymers $\mathrm{F} 127,5.00 \mathrm{~g}$ of $\mathrm{KCl}, 2.0$ of 1,3,5-trimethylbenzene (TMB) were dissolved in $120 \mathrm{ml}$ of $2.0 \mathrm{M} \mathrm{HCl}$ aqueous solution with stirring or static at different temperatures for $24 \mathrm{~h}$. Then $8.3 \mathrm{~g}$ of tetraethylorthosilicate (TEOS) as the silicate source was added dropwise into the above surfactant solution for another $24 \mathrm{~h}$. Afterwards the mixture was transferred into a Teflon bottle and heated statically at $100^{\circ} \mathrm{C}$ for $48 \mathrm{~h}$. The final as-synthesized product was obtained by filtering, washing with distilled water, and drying at $353 \mathrm{~K}$ for $24 \mathrm{~h}$ in air, ultimately, calcined at $823 \mathrm{~K}$ for $6 \mathrm{~h}$. The samples were denoted as $\mathrm{Sc} / \mathrm{Sg}$-x, where $\mathrm{x}$ is the temperature 
of water bath, $\mathrm{x}=25,30,35,40,45$. For example, Sc-30 denotes a sample synthesized at $30^{\circ} \mathrm{C}$ water bath under static condition.

\section{Characterization}

The samples were characterized by small-angle $X$ ray powder diffraction (XRD), scanning electron microscopy (SEM), transmission electron microscopy (TEM) and $\mathrm{N}_{2}$ adsorption-desorption. Low-angle XRD patterns were performed between $0.5^{\circ}-5^{\circ}$ on an instrument (Shimadzu X-6000) at $40 \mathrm{kV}$ using $\mathrm{Cu} \mathrm{Ka}\left(\mathrm{k}=0.154 \mathrm{~nm}\right.$, step size $\left.0.02^{\circ}, 0.5 \mathrm{~s}\right)$ radiation. The SEM images were obtained on a Cambridge S-360 apparatus operating at $20 \mathrm{kV}$. The samples were coated with gold before the experiment. The TEM images were obtained by using a JEOL JEM 2100 electron microscope equipped with a field emission source at an accelerating voltage of $200 \mathrm{Kv} . \mathrm{N}_{2}$ adsorption-desorption measurements were carried out using a Micromeritics TriStar II 2020 porosimetry analyzer. Before the measurement, all the samples have degassed for $8 \mathrm{~h}$. The specific surface area $\left(\mathrm{S}_{\mathrm{BET}}\right)$ was calculated via the Brunauer-Emmett-Teller (BET) method. The total pore volumes $\left(\mathrm{V}_{\mathrm{P}}\right)$ were calculated from the amounts of nitrogen adsorbed at a relative pressure of approximately $\mathrm{P} / \mathrm{P}_{0}=0.99$.

\section{Results and discussion}
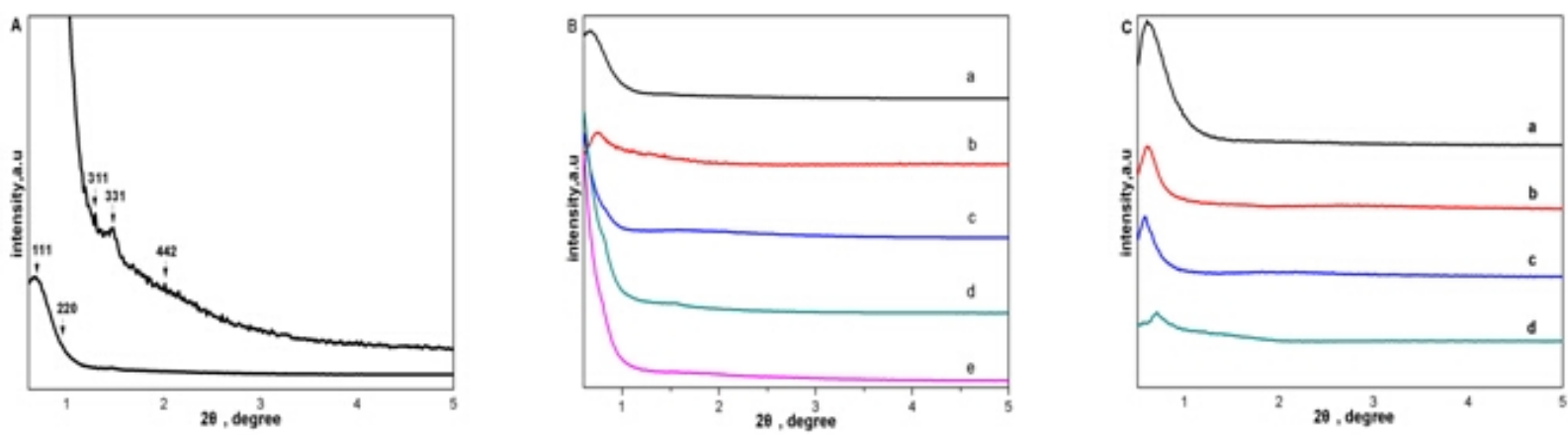

Fig.1 (A) XRD pattern of FDU-12; (B) XRD patterns of (a) Sg-25; (b) Sg-30; (c) Sg-35; (d) Sg-40; (e) Sg-45; (C) XRD patterns of (a) Sc-30; (b) Sc-35; (c) Sc-40; (d) Sc-45.

The low-angle XRD patterns of all samples are shown in Fig.1A. All samples exhibit five characteristic peaks, indexed as (111), (220), (311), (331) and (442) reflections respectively as described in the literature[3], proving that they all have the structural characteristic(face-centred cubic structure Fm3m) of the mesoporous silica FDU-12. As can be seen clearly from the Fig.1B and Fig.1C, the peak intensity of all samples weaken with the increasing water-bath temperature due to the reduction in crystallinity which is in accordance with the TEM results.
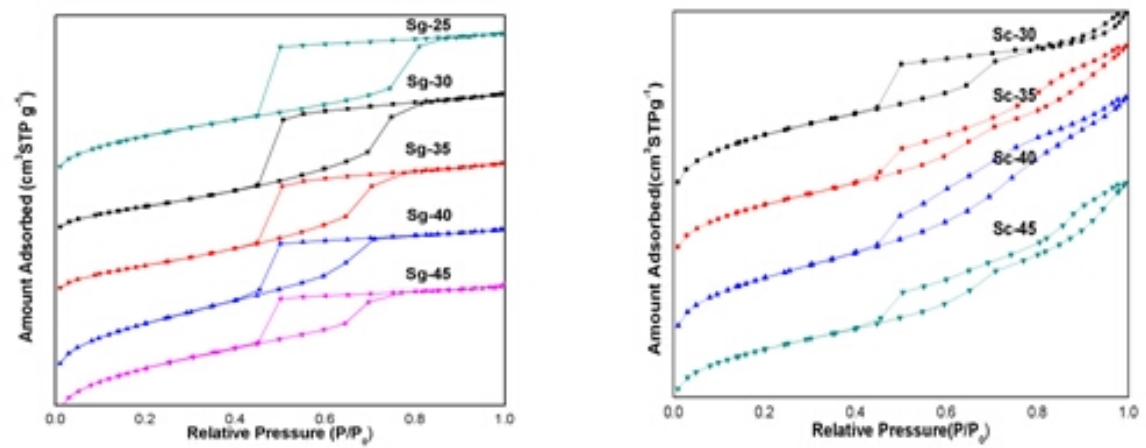

Fig. $2 \mathrm{~N}_{2}$ adsorption-desorption isotherms of different samples 
Table 1. Texture parameters of all samples with different synthetic conditions

\begin{tabular}{cccc}
\hline Sample & $\begin{array}{c}\text { BET } \\
\text { surface } \\
\text { area }\end{array}$ & $\begin{array}{c}\text { Total Pore } \\
\text { Volume } \\
{\left[\mathrm{cm}^{3} \cdot \mathrm{g}^{-1}\right]}\end{array}$ & $\begin{array}{c}\text { Pore diameter } \\
{[\mathrm{nm}]^{\mathrm{a}}}\end{array}$ \\
\hline Sg-25 & 470 & 0.53 & 5.10 \\
Sg-30 & 503 & 0.54 & 6.08 \\
Sg-35 & 539 & 0.46 & 5.13 \\
Sg-40 & 632 & 0.59 & 4.05 \\
Sg-45 & 637 & 0.52 & 4.02 \\
Sc-30 & 587 & 0.52 & 4.56 \\
Sc-35 & 514 & 0.61 & 5.53 \\
Sc-40 & 599 & 0.69 & 5.57 \\
Sc-45 & 470 & 0.62 & 5.92 \\
\hline
\end{tabular}

$\mathrm{N}_{2}$ adsorption-desorption isotherms of different samples are shown in Fig.2. Mesoporous pure silica FDU-12 synthesized under stirring conditions shows a larger $\mathrm{H} 2$ hysteresis loop. Nevertheless, when synthesized under static conditions, mesoporous silica FDU-12 shows a H3 or H4 hysteresis loop due to the shape of slit pore structure. The pore structure parameters of different samples are listed in Table 1.
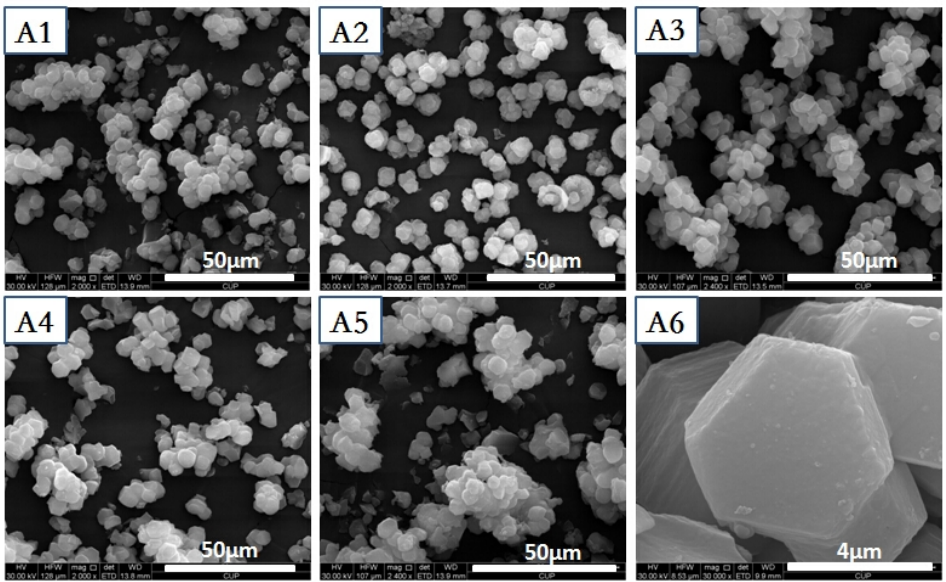

Fig.3A The SEM images of different samples (A1) Sg-25; (A2) Sg-30; (A3) Sg-35; (A4) Sg-40; (A5) Sg-45.
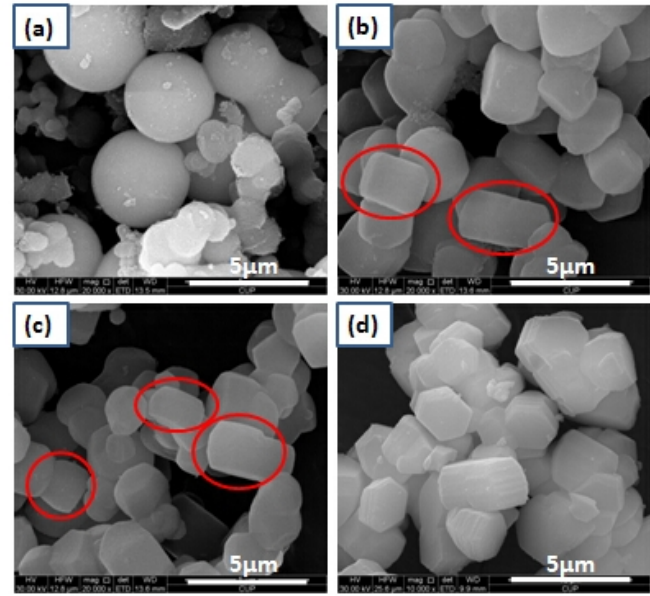

Fig.3B The SEM images of different samples (a)Sc-30; (b) Sc-35; (c) Sc-40; (d) Sc-45. 
The SEM image of FDU-12 under static condition is given in Fig.3B. It is found that FDU-12-Sc-30 shows a spherical morphology, as shown in Fig.3B(b) and Fig.3B(c), The samples (FDU-12 Sc-35、Sc-40) show a mixture of spherical and flake cuboid morphology, then change into regular hexagonal prism at $45^{\circ} \mathrm{C}$. Thus, we can project that the morphology of FDU-12 is gradually changed from the spherical morphology to flake cuboid to regular hexagonal prism with increasing of the water-bath temperature.

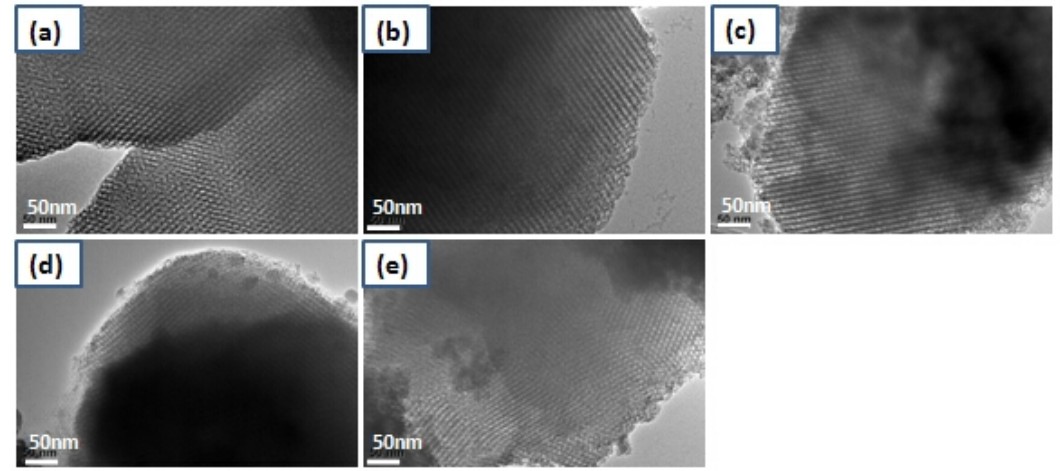

Fig.4 The TEM images of different samples (a) Sg-30; (b) Sc-30; (c) Sc-35; (d) Sc-40; (e) Sc-45.

The TEM images of FDU-12 are shown in Fig.4. Under TEM observations, all the FDU-12 products synthesized under different conditions remain the well-ordered structures. Moreover, degree of ordering will decrease with the increasing of the water-bath temperature.

\section{Conclusions}

By simply changing the water-bath temperature, mesoporous FDU-12 silicas with different morphologies were synthesized. Under static condition, the spherical morphology can be obtained at $30^{\circ} \mathrm{C}$ water-bath temperature and a flake cuboid morphology will appear when synthesized in the 30 ${ }^{\circ} \mathrm{C}$ water-bath. Under Static $45^{\circ} \mathrm{C}$ and stirring conditions, the morphologies exhibit almost the hexagonal prism. This simple synthetic strategy can be extended to synthesize many related materials. Meanwhile, FDU-12 mesoporous materials with both excellent structural parameters and different morphologies will create a good prospect for the future application in the extensive fields, such as catalysis, adsorption, biological separation, and others.

\section{Acknowledgement}

This work was supported by National Natural Science Foundation of China (No. 21276277 and U1463207), CNOOC HuizhouRefinery Branch(HL00FW2012-0196), National Basic Research Program of China (Grant No. 2012CB215001 \& 2012CB215002), and CNPC major project.

\section{References}

[1] Ding Z, Chen J, Gong X. Effect of different kinds of acid and their concentration on mesostructure and morphology of ordered mesoporous molecular sieve SBA-15[J]. Journal of the Chinese Ceramic Society, 2008, 36(7): 978-984.

[2] Zhou L, Zhang L, Liu H. Effect of crystallization temperature on structure and morphology of mesoporous SBA-15[J]. Chinese Journal of Process Engineering, 2006, 6(3): 499.

[3] Fan J, Yu C, Gao F, et al. Cubic mesoporous silica with large controllable entrance sizes and advanced adsorption properties[J]. Angewandte Chemie, 2003, 115(27): 3254-3258. 